\title{
Formation of the Nitrative DNA Lesion 8-Nitroguanine is Associated with Asbestos Contents in Human Lung Tissues: A Pilot Study
}

\author{
Yusuke Hiraku ${ }^{1}$, Kiyoshi SaKaI ${ }^{2}$, Eiji Shibata ${ }^{3}$, Michihiro Kamisima ${ }^{4}$, \\ Naomi Hisanaga ${ }^{5}$, Ning MA $^{6}$, Shosuke KawANISHI ${ }^{7}$ and Mariko Murata ${ }^{1}$
}

${ }^{1}$ Department of Environmental and Molecular Medicine, Mie University Graduate School of Medicine, Japan, ${ }^{2}$ Nagoya City Public Health Research Institute, Japan, ${ }^{3}$ Department of Health and Psychosocial Medicine, Aichi Medical University School of Medicine, Japan, ${ }^{4}$ Department of Occupational and Environmental Health, Nagoya City University Graduate School of Medical Sciences, Japan, ${ }^{5}$ Center for Campus Health and Environment, Aichi University of Education, Japan, ${ }^{6}$ Faculty of Health Science, Suzuka University of Medical Science, Japan and ${ }^{7}$ Faculty of Pharmaceutical Sciences, Suzuka University of Medical Science, Japan

\begin{abstract}
Formation of the Nitrative DNA Lesion 8-Nitroguanine is Associated with Asbestos Contents in Human Lung Tissues: A Pilot Study: Yusuke HIRAKU, et al. Department of Environmental and Molecular Medicine, Mie University Graduate School of Medicine-Objectives: Asbestos causes lung cancer and malignant mesothelioma, and chronic inflammation is considered to participate in carcinogenesis. However, biomarkers to evaluate its carcinogenic risk have not been established. Reactive oxygen/nitrogen species are generated in biological systems under inflammatory conditions and may contribute to carcinogenesis by causing DNA damage. In this study, we examined the relationship between the formation of 8nitroguanine (8-nitroG), a mutagenic DNA lesion formed during inflammation, and asbestos contents in human lung tissues. Methods: We obtained non-tumor lung tissues from patients with $(n=15)$ and without mesothelioma $(n=21)$. The expression of 8 -nitroG and related molecules was examined by immunohistochemistry, and their staining intensities were semiquantitatively evaluated. Asbestos contents in lung tissues were analyzed by analytical transmission electron microscopy. Results: In subjects without mesothelioma, staining intensities of 8-nitroG and apurinic/apyrimidinic endonuclease 1 (APE1) were significantly correlated with total asbestos and amphibole contents $(p<0.05)$, but not with chrysotile content. In mesothelioma patients,
\end{abstract}

Received Sept 26, 2013; Accepted Jan 22, 2014

Published online in J-STAGE Mar 4, 2014

Correspondence to: Y. Hiraku, Department of Environmental and Molecular Medicine, Mie University Graduate School of Medicine, Tsu, Mie 514-8507, Japan (e-mail: y-hiraku@doc.medic.mie-u.ac. jp) their staining intensities were not correlated with asbestos contents. The double immunofluorescence technique revealed that APE1 was expressed in 8-nitroGpositive cells, suggesting that abasic sites were formed possibly due to the removal of 8-nitroG. The staining intensities of 8-oxo-7,8-dihydro-2'-deoxyguanosine, an oxidative DNA lesion, and its repair enzyme 8-oxoguanine DNA-glycosylase were correlated with age $(p<0.05)$, but not with asbestos contents in subjects without mesothelioma. Conclusions: This is the first study to demonstrate that 8-nitroG formation is associated with asbestos contents in human lung tissues. This finding raises a possibility that 8-nitroG serves as a biomarker that can be used to evaluate asbestos exposure and carcinogenic risk.

(J Occup Health 2014; 56: 186-196)

Key words: 8-Nitroguanine, Asbestos, Carcinogenesis, DNA damage, Lung, Reactive nitrogen species

Asbestos is a naturally occurring fiber causing lung cancer and malignant mesothelioma of the pleura and peritoneum. The International Agency for Research on Cancer (IARC) has classified asbestos as carcinogenic to humans (Group 1) ${ }^{1}$. Amphiboles, including crocidolite and amosite, are more potent carcinogens than chrysotile ${ }^{1)}$. Although possible mechanisms for asbestos-induced carcinogenesis have been proposed ${ }^{2}$, the precise mechanism remains to be clarified. In addition, there are no established biomarkers to evaluate the risk of asbestos-induced carcinogenesis.

Chronic inflammation is induced by a wide variety of physical and chemical agents, including asbestos, and has been postulated to be an important risk factor 
of cancer $^{3,4)}$. Reactive oxygen (ROS) and nitrogen species (RNS) are generated from inflammatory and epithelial cells under such conditions and are considered to participate in carcinogenesis by causing DNA damage $^{5,6}$. 8-Nitroguanine (8-nitroG) is a mutagenic DNA lesion formed during chronic inflammation via the reaction of guanine with peroxynitrite $\left(\mathrm{ONOO}^{-}\right)$, which is formed by the interaction of nitric oxide (NO) with superoxide anion $\left(\mathrm{O}_{2}^{\cdot-}\right)^{7,8)}$. We have previously demonstrated that 8 -nitroG is formed in tissues at a precancerous stage in patients with cancer-prone inflammatory diseases, including cervical dysplasia caused by human papilloma virus ${ }^{9)}$ and nasopharyngitis mediated by Epstein-Barr virus ${ }^{10)}$. We have reported that intratracheal instillation of asbestos induced 8-nitroG formation in lung tissues of mice and that crocidolite induced significantly stronger DNA damage than chrysotile, which is consistent with their carcinogenic potentials ${ }^{11)}$. However, the association of 8-nitroG formation with asbestos contents in human lung tissues has not been investigated.

In this study, we performed immunohistochemical analysis to examine 8-nitroG formation in human lung tissues. The contents of asbestos in lung tissues were quantitatively analyzed by analytical transmission electron microscopy. The correlation between asbestos contents and staining intensity of 8-nitroG was statistically analyzed to examine whether 8-nitroG could be used as a biomarker to evaluate asbestos exposure and carcinogenic risk. We also examined the expression of inducible NO synthase (iNOS) and apurinic/apyrimidinic endonuclease 1 (APE1), which participates in base excision repair of an abasic site ${ }^{12)}$. Because the glycosidic bond between 8-nitroG and deoxyribose is relatively unstable, 8-nitroG may be released from DNA to form an abasic site ${ }^{13)}$. In addition, the formation of 8-oxo-7,8-dihydro-2'-deoxyguanosine (8-oxodG), an oxidative DNA lesion, and the expression of its repair enzyme 8-oxoguanine DNA-glycosylase (OGG1) ${ }^{14}$ ) were investigated in a similar manner in comparison with 8-nitroG.

\section{Materials and Methods}

\section{Subjects and lung tissues}

Non-tumor lung tissues $(n=36)$ were obtained from autopsy and surgical specimens of 15 patients with malignant mesothelioma ( 9 males and 6 females, age $60.7 \pm 11.9$ years, mean \pm SD) and 20 subjects (21 specimens) without mesothelioma (11 males and 9 females, age $66.3 \pm 9.2$ years). Tissue specimens were resected at 16 hospitals from 1980 to 2008 . The diagnosis of malignant mesothelioma was made as described previously ${ }^{15)}$. Cases of asbestos-related diseases, such as lung cancer and asbestosis, were not included as subjects without mesothelioma. There was no significant difference in age between these groups ( $p=0.128$ by Student's $t$-test). The characteristics of each subject are listed in Table S1. Smoking habit was not recorded in most mesothelioma cases because smoking is not considered to be a risk factor of this disease $\mathrm{e}^{4}$. Because this study was conducted in a retrospective manner with historical materials, we could not obtain written informed consent. Identifying information was removed from all samples before analysis for strict privacy protection. This study was performed in accordance with the Ethical Guidelines for Epidemiological Research enacted by the Japanese Government and was approved by the Ethical Committee of Mie University Graduate School of Medicine, Japan (No. 1078). Information concerning occupational history and smoking habit was obtained from personal or telephone interview of the subjects or their relatives and hospital records as described previously ${ }^{15}$.

\section{Analysis of asbestos contents in lung tissues}

The contents of asbestos (chrysotile and amphiboles) and non-asbestos fibers in lung tissues were analyzed by analytical transmission electron microscopy as described previously ${ }^{15)}$. Tissue specimens were preserved in $10 \%(\mathrm{v} / \mathrm{v})$ formaldehyde solution. Then, the tissues were freeze-dried and subsequently ashed in a low-temperature plasma asher (LTA-2S, Yanagimoto, Kyoto, Japan) for 24 hours. The ashes were suspended in distilled water and treated in an ultrasonic bath (Branson 3200, Yamato, Tokyo, Japan) for 5 minutes. The suspended ashes were filtered through a $0.2 \mu \mathrm{m}$ Nuclepore filter (Nuclepore Corp., Pleasanton, CA, USA). The filters with deposits were coated with a thin layer of carbon in a sputtering device (JEE-4X, JEOL, Akishima, Japan). Random sections of the filters were transferred to carbon-coated nickel grids (200 mesh, Veco, Eerbeek, Netherlands), and chloroform was added to dissolve the filters. Samples were scanned at a magnification of $\times 10,000$ on an analytical transmission electron microscope (H800, Hitachi, Tokyo, Japan, or JEM 2010, JEOL). Only particles with approximately parallel sides and an aspect ratio of 3 or more were considered as inorganic fibers. Asbestos was morphologically identified with the help of an energy-dispersive X-ray analyzer (7000Q, Kevex, Foster City, CA, USA, or NORAN System SIX 300, Thermo Electron, Middleton, WI, USA). Asbestos types were determined by comparing the elemental compositions with those of standard reference samples obtained from the Japan Asbestos Association. The other types of fibers were regarded as non-asbestos fibers. The shortest and thinnest fibers were $0.2 \mu \mathrm{m}$ in length and $0.02 \mu \mathrm{m}$ in diameter. The fiber content (per gram of dry lung) 
was calculated from the number of fibers counted, and the weight of the dry lung used in the analysis. We also calculated the number of fibers with a length of $1 \mu \mathrm{m}$ or more to examine the effect of fiber length on DNA damage. When the number of fibers was smaller than the limit of quantitation, the fiber content was set at half of this limit, which was dependent on tissue weight and the number of grid openings analyzed $^{15)}$.

Immnunoperoxidase staining and evaluation of staining intensity

The formation of DNA lesions (8-nitroG and 8-oxodG) and the expression of iNOS and DNA repair enzymes (OGG1 and APE1) in lung tissues were assessed by immunoperoxidase staining. We produced rabbit polyclonal anti-8-nitroG antibody as described previously ${ }^{16)}$. Lung tissues adjacent to those used for fiber analysis were embedded in paraffin and sectioned. The tissue sections ( $6 \mu \mathrm{m}$ thick) were deparaffinized in xylene, rehydrated in ethanol and then microwaved in $5 \%(\mathrm{w} / \mathrm{v})$ urea for antigen retrieval. Endogenous peroxidase was quenched by $0.3 \%(\mathrm{v} / \mathrm{v})$ hydrogen peroxide $\left(\mathrm{H}_{2} \mathrm{O}_{2}\right)$ for 60 minutes, followed by the treatment with $1 \%(\mathrm{w} / \mathrm{v}) \mathrm{Difco}^{\mathrm{TM}}$ skim milk (Becton Dickinson and Company, Sparks, MD, USA) in PBS for 60 minutes at room temperature. Then, the sections were incubated with the primary antibody [rabbit polyclonal anti-8-nitroG antibody $(0.5 \mu \mathrm{g} / \mathrm{m} l)$, mouse monoclonal anti-8-oxodG antibody (clone $\mathrm{N} 45.1,0.5 \mu \mathrm{g} / \mathrm{m} l$, Japan Institute for the Control of Aging, Fukuroi, Japan), mouse monoclonal anti-iNOS antibody (clone NOS-IN, 1:300, Sigma, St. Louis, MO, USA), rabbit polyclonal anti-OGG1 antibody (ab22766, 1:250, Abcam, Cambridge, UK) or mouse monoclonal anti-APE1 antibody (ab3722, 1:100, Abcam)] overnight at room temperature. The sections treated with rabbit polyclonal antibody were then incubated with goat anti-rabbit IgG antibody (1:100, Santa Cruz Biotechnology, Santa Cruz, CA, USA) overnight, followed by treatment with peroxidase-antiperoxidase complex for 2 hours at room temperature. The sections treated with mouse monoclonal antibody were then treated with horseradish peroxidase-conjugated goat anti-mouse IgG antibody (1:100, Santa Cruz Biotechnology) for 2 hours at room temperature. The immunostaining was developed by using $0.1 \mathrm{mg} / \mathrm{ml}$ of 3,3'-diaminobenzidine tetrahydrochloride (Dojindo Laboratories, Kumamoto, Japan) as a chromogen in PBS containing $0.03 \%(\mathrm{v} / \mathrm{v}) \mathrm{H}_{2} \mathrm{O}_{2}$. Then, the sections were observed under a light microscope, and the staining intensity of each field was semiquantitatively evaluated and given a score of 0 (negative), 1 (moderately positive) or 2 (strongly positive). Then, we calculated the average of the score throughout the section of each subject (approximately 14 fields on average) as a continuous variable, which was used for statistical analysis.

\section{Double immunofluorescence study}

To examine the colocalization of DNA lesions and repair enzymes, a double immunofluorescence study was performed as described previously9). Paraffin sections were deparaffinized, microwaved and then incubated with the primary antibodies [(a) rabbit polyclonal anti-8-nitroG antibody $(1 \mu \mathrm{g} / \mathrm{m} l)$ plus mouse monoclonal anti-8-oxodG antibody $(0.5 \mu \mathrm{g} / \mathrm{ml})$, (b) mouse monoclonal anti-8-oxodG antibody plus rabbit polyclonal anti-OGG1 antibody (1:200) or (c) rabbit polyclonal anti-8-nitroG antibody plus mouse monoclonal anti-APE1 antibody (1:100)] overnight at room temperature. Then, the sections were incubated with fluorescent secondary antibodies [Alexa 594-labeled goat antibody against rabbit $\mathrm{IgG}$ and Alexa 488-labeled goat antibody against mouse IgG (1:400 each, Molecular Probes Inc., Eugene, OR, USA)] for 3 hours at room temperature. The stained sections were examined under a fluorescent microscope (BX53, Olympus, Tokyo, Japan). After observation, these sections were stained with hematoxylin and eosin.

\section{Statistical analysis}

The contents of chrysotile, amphiboles and non-asbestos fibers showed a log-normal distribution (confirmed by chi-square goodness of fit test). Thus we used log-transformed values of fiber contents for statistical analysis. We compared fiber contents or staining intensities in lung tissues of the two different groups by Student's $t$-test. The correlation of staining intensity and fiber contents was analyzed by Pearson's correlation test. The correlation of staining intensity and duration of occupational asbestos exposure was analyzed by Spearman's correlation test. Multiple stepwise regression analysis was performed to determine which variables (contents of chrysotile, amphiboles and non-asbestos fibers, age and smoking habit) predict the extent of 8-nitroG and 8-oxodG formation. The statistical analysis was performed with IBM SPSS Statistics software version 20 for Macintosh. $p$ values less than 0.05 were considered to be statistically significant.

\section{Results}

\section{Inorganic fiber contents in lung tissues}

Clinical and occupational information of subjects who participated in this study is listed in Table $\mathrm{S} 1$. The difference in the contents of asbestos and non-asbestos fibers in lung tissues of subjects with and without mesothelioma is shown in Fig. 1. In mesothelioma patients, the contents of total inorganic 
fibers (asbestos plus non-asbestos fibers) [geometric mean $\pm \mathrm{SD}, 87.28 \pm 3.20\left(\times 10^{6}\right.$ fibers/g dry lung $)$, $p<0.05$ ] and total asbestos (chrysotile plus amphiboles) $(13.45 \pm 5.27, p<0.001)$ in lung tissues were significantly larger than those in subjects without mesothelioma $(35.16 \pm 2.37$ and $1.94 \pm 2.76$, respectively). The contents of chrysotile and amphiboles were also significantly larger in mesothelioma patients $(3.77 \pm 5.85$ and $7.39 \pm 6.27$, respectively) than in subjects without mesothelioma $(0.60 \pm 2.24$ and $1.14 \pm 3.61$, respectively) $(p<0.01)$. The content of inorganic fibers in subjects with mesothelioma $(62.37 \pm 3.07)$ was larger than that in those without mesothelioma $(32.37 \pm 2.40)$, although the difference was not statistically significant $(p=0.057)$ (Fig. 1A). When the fiber length was limited to $\geq 1 \mu \mathrm{m}$, the contents of all types of fibers in mesothelioma patients were significantly larger than those in subjects without mesothelioma $(p<0.05)$ (Fig. 1B). There was no significant difference in the contents of any type of inorganic fibers between males and females (Fig. S1A) or between smokers and nonsmokers without mesothelioma (Fig. S1B).

\section{DNA damage and expression of related molecules}

To examine the intensity and the location of DNA lesions and related molecules, we performed immunoperoxidase staining. DNA lesions (8-nitroG and 8-oxodG) were formed in alveolar and bronchial epithelial cells and inflammatory cells (Fig. 2A). DNA lesions were also observed in mesothelial cells of some tissue specimens (Fig. 2A). Representative images of tissue sections negative (score of 0 ), moderately positive (score of 1) and strongly positive (score of 2) for these molecules are shown in Fig. 2B. The average score throughout the section of each subject was calculated and used for statistical analysis. To investigate the colocalization of DNA lesions and repair enzymes, we performed double immunofluorescent staining. The representative images are shown in Fig. 3. 8-NitroG and 8-oxodG were colocalized in the nucleus (Fig. 3A). OGG1 expression was observed in 8-oxodG-positive cells (Fig. 3B). APE1 expression was partially seen in 8-nitroG-positive cells (Fig. 3C).

Correlation of staining intensities of DNA lesions and related molecules

We analyzed the correlation of staining intensities of DNA lesions with those of related molecules. Scatter diagrams of their staining intensities are shown in Fig. 4. The staining intensity of 8-nitroG was significantly correlated with those of 8-oxodG $(p=0.009)$, iNOS $(p<0.001)$ and APE1 $(p<0.001)$. The staining intensity of 8-oxodG was significantly correlated with that of OGG1 $(p<0.001)$.

Difference in staining intensities of lung tissues The difference in staining intensities of DNA
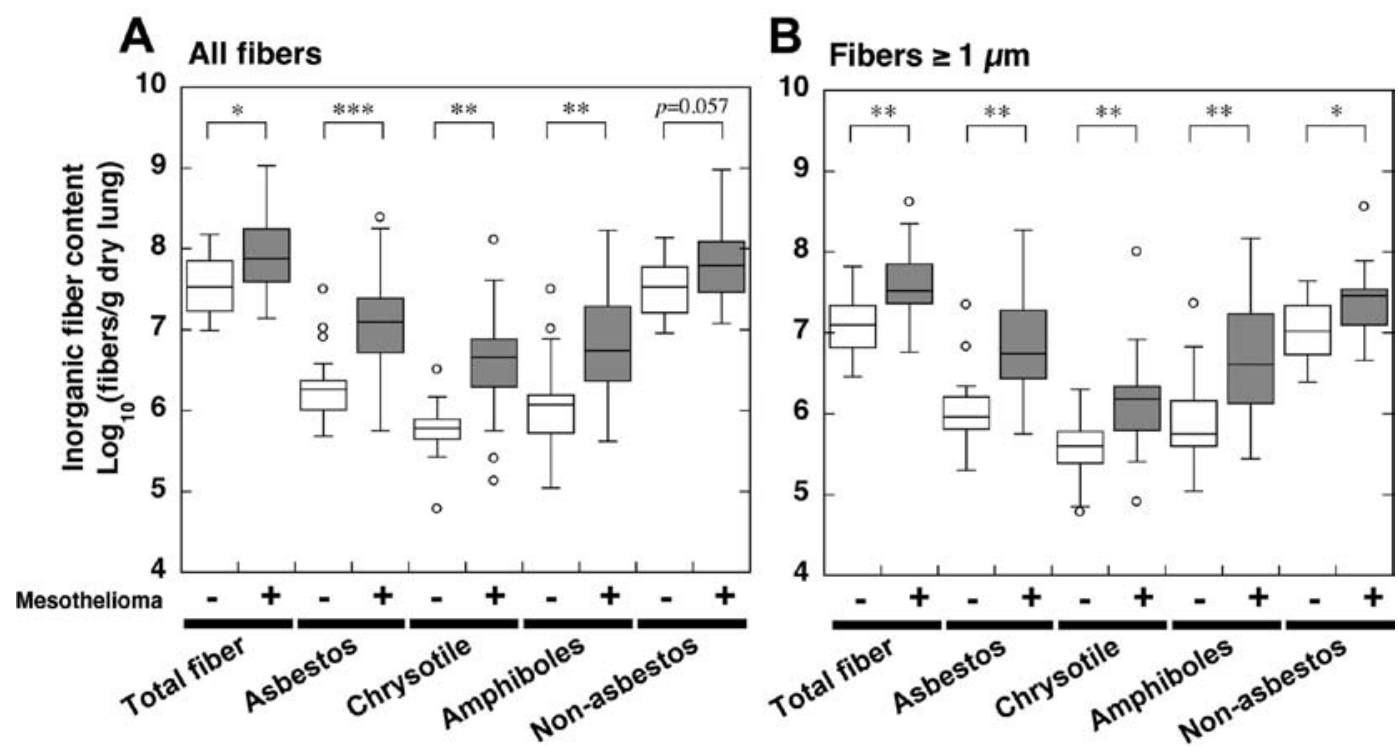

Fig. 1. Difference in inorganic fiber contents in lung tissues of subjects with and without malignant mesothelioma. The contents of asbestos (chrysotile and amphiboles) and non-asbestos fibers in lung tissues [all fibers (A) and fibers with a length of $\geq 1 \mu \mathrm{m}$ (B)] were analyzed as described in Materials and Methods. The difference in the number of these fibers in subjects without $(-, n=21)$ and with malignant mesothelioma $(+, \mathrm{n}=15)$ was analyzed by Student's $t$-test. Total fiber (total inorganic fiber) $=$ asbestos + non-asbestos fiber; asbestos $=$ chrysotile + amphiboles. ${ }^{*} p<0.05 ; * * p<0.01 ; * * *<0.001$. 
A
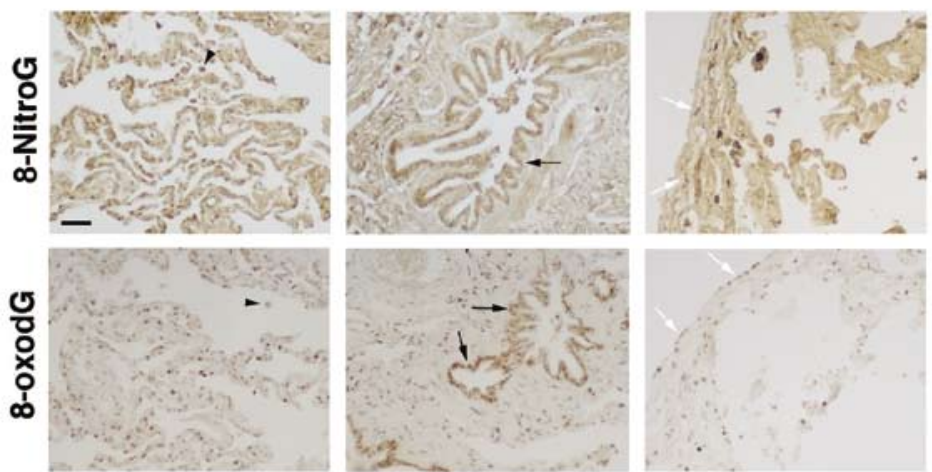

B
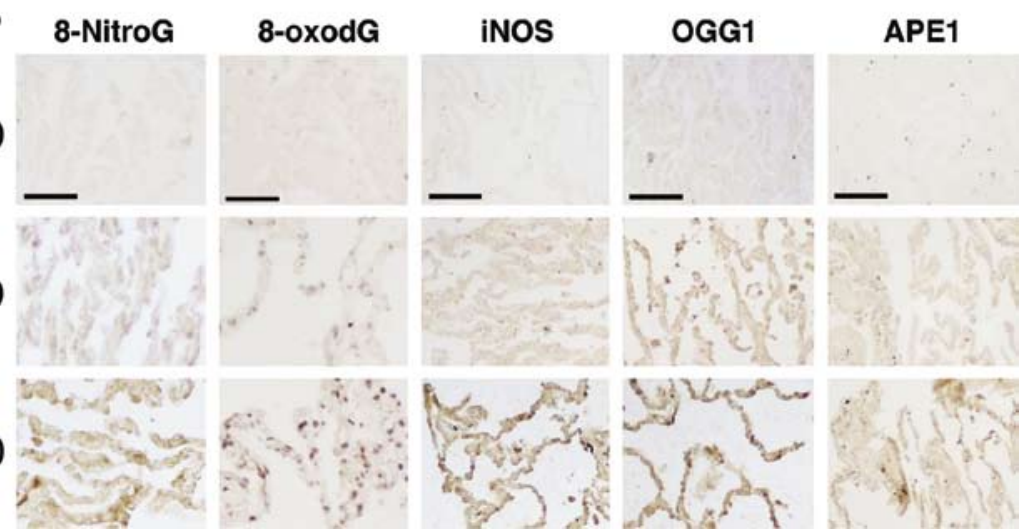

(+)

$(++)$
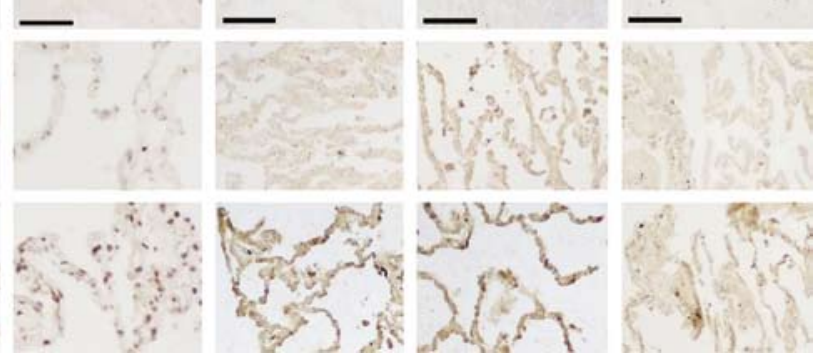

A
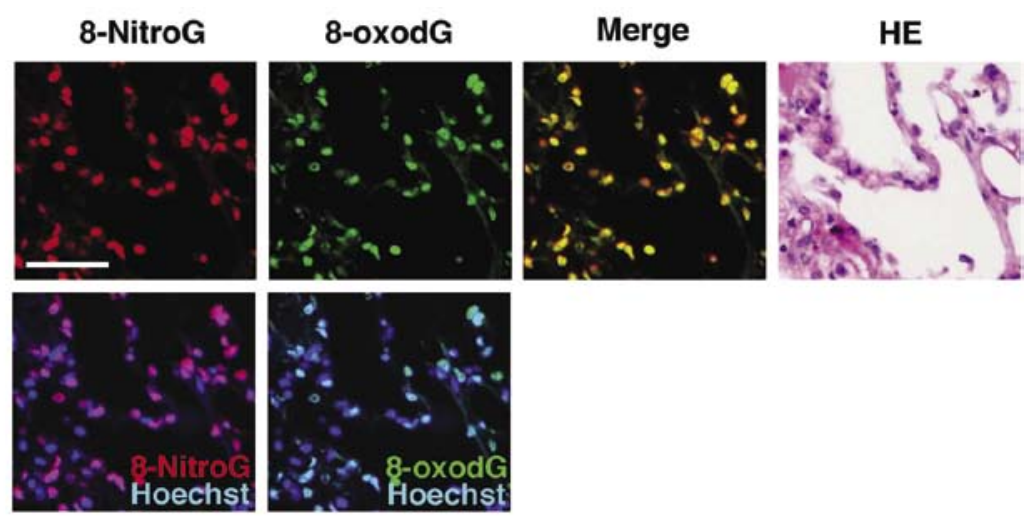

B

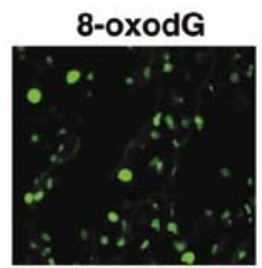

C
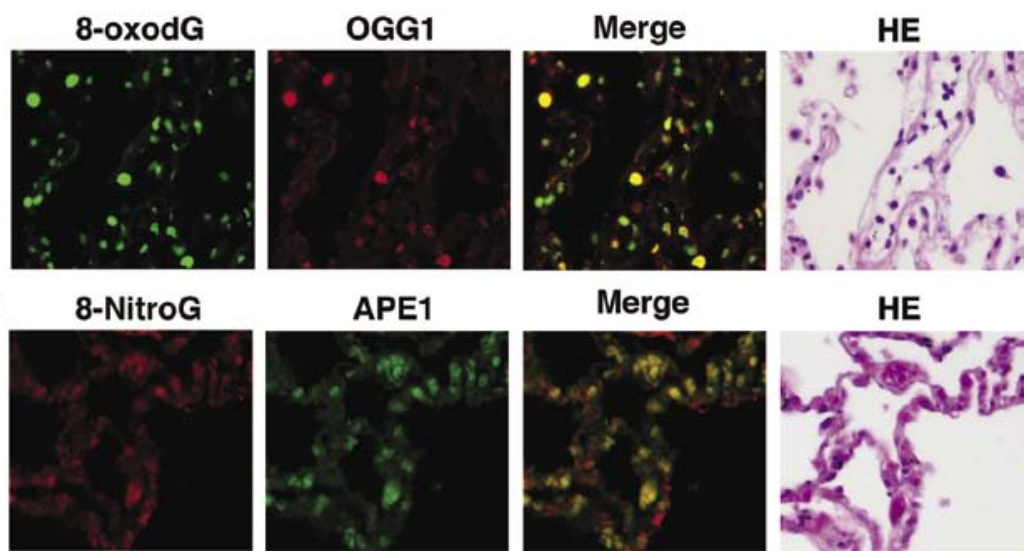

Merge

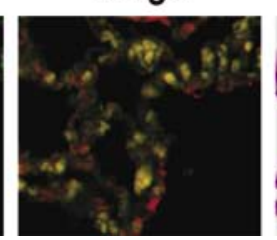

Fig. 2. Immunoperoxidase staining of DNA lesions and related molecules in lung tissues. DNA lesions (8-nitroG and 8-oxodG), iNOS and DNA repair enzymes (OGG1 and APE1) were immunostained as described in Materials and Methods. (A) Representative images of lung tissues with intensive DNA damage. 8-NitroG and 8-oxodG formation was observed in inflammatory cells (left, arrowheads, case 10 in Table S1), alveolar epithelial cells, bronchial epithelial cells (middle, black arrows, case 10) and mesothelial cells (right, white arrows, case 14). Scale bar $=50 \mu \mathrm{m}$. (B) Representative images of negatively and positively stained tissues. Staining intensity of these images was evaluated as negative $(-$, score of 0$)$, moderately positive $(+$, score of 1$)$ or strongly positive $(++$, score of 2). Scale bar $=50 \mu \mathrm{m}$ (8-nitroG and 8-oxodG) or $100 \mu \mathrm{m}$ (iNOS, OGG1 and APE1).

Fig. 3. Double immunofluorescent staining of DNA lesions and repair enzymes in lung tissues. The formation of DNA lesions and the expression of DNA repair enzymes were analyzed by double immunofluorescent staining as described in Materials and Methods. (A) Formation of DNA lesions. 8-NitroG (red) and 8-oxodG (green) were formed in the nuclei of epithelial cells and colocalized (merge, yellow, case 13 in Table $\mathrm{S} 1)$. The tissue was treated with $5 \mu \mathrm{M}$ Hoechst 33258 to stain the nuclei (blue). (B) 8-oxodG formation and OGG1 expression. OGG1 expression (red) was observed in 8-oxodG-positive cells (green, case 13). (C) 8-NitroG formation and APE1 expression. APE1 expression (green) was partially observed in 8-nitroG-positive cells (red, case 8). The immunostained sections were then stained with hematoxylin and eosin (HE). Scale bar $=50 \mu \mathrm{m}$. 

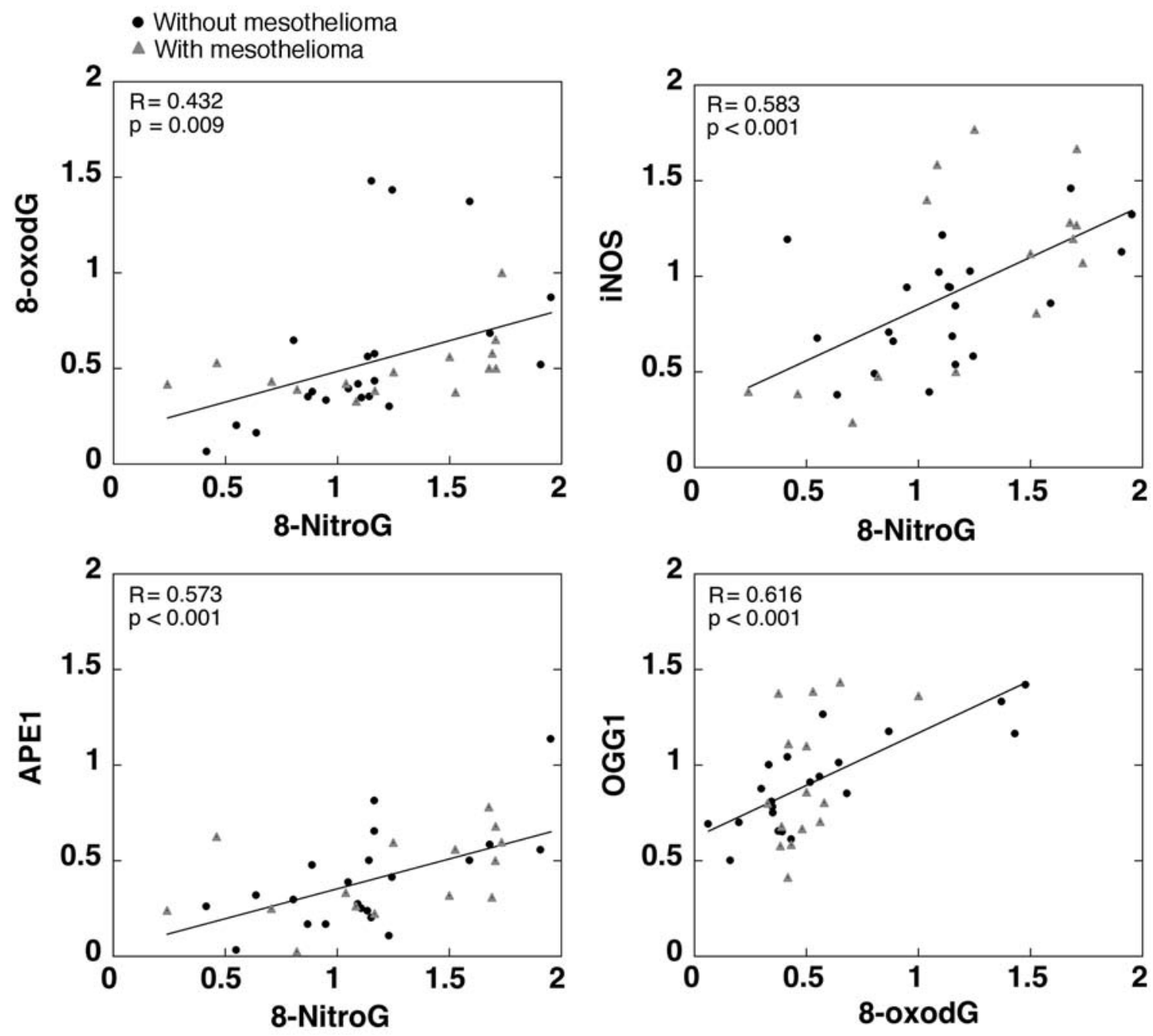

Fig. 4. Correlation of staining intensities of DNA lesions and related molecules in lung tissues. Staining intensities of DNA lesions (8-nitroG and 8-oxodG), iNOS and DNA repair enzymes (OGG1 and APE1) were evaluated and calculated as described in Materials and Methods. The correlation of their staining intensities was analyzed by Pearson's correlation test. Black circle and gray triangle, subjects without $(\mathrm{n}=21)$ and with malignant mesothelioma $(n=15)$, respectively.

lesions and repair enzymes in lung tissues between patients with and without mesothelioma is shown in Fig. S2. Although the staining intensity of 8-nitroG tended to be slightly higher in mesothelioma patients, there was no significant difference in staining intensities of any molecules in these groups. These was no significant difference in their staining intensities in males and females, although iNOS immunoreactivity was significantly higher in males (Fig. S3A). There was no significant difference in staining intensities between smokers and nonsmokers without mesothelioma (Fig. S3B).

\section{Correlation of staining intensities with fiber contents}

We performed statistical analysis to examine the correlation of the staining intensity of DNA lesions and related molecules with inorganic fiber contents in lung tissues. Scatter diagrams of asbestos contents and the staining intensities of DNA lesions are shown in Fig. 5. The staining intensity was not correlated with any types of inorganic fibers in all cases (Table S2). However, interestingly, in subjects without mesothelioma, the staining intensity of 8-nitroG was significantly correlated with the total asbestos $(R=0.560$, $p=0.008)$ and amphibole contents $(R=0.534, p=0.013)$ (Fig. 5 and Table $\mathrm{S} 2$ ). The staining intensity of APE1 was also significantly correlated with the contents of total asbestos $(R=0.582, p=0.006)$ and amphiboles $(R=0.530, p=0.013)$. The staining intensities of 8-oxodG, iNOS and OGG1 tended to correlate with 

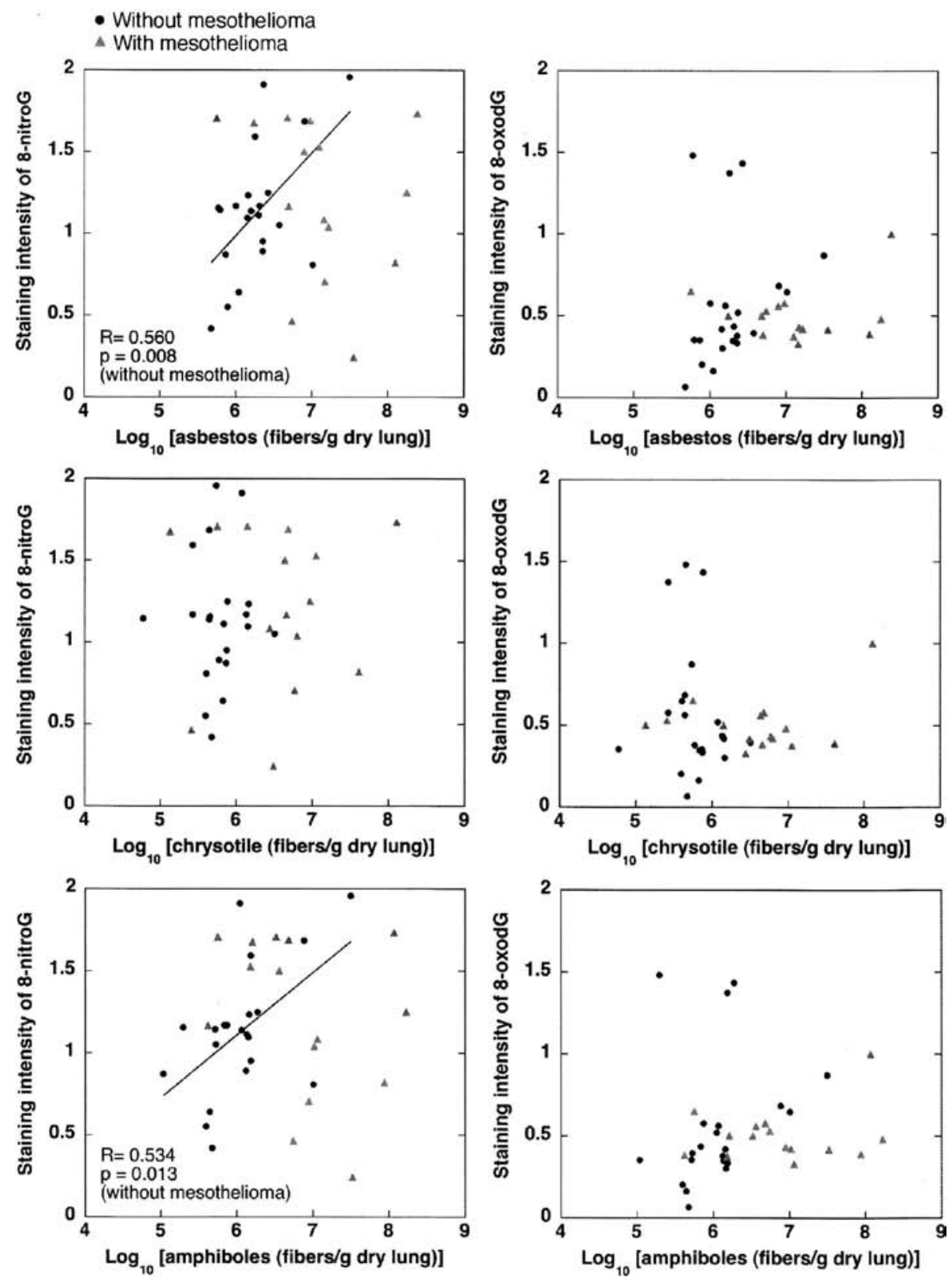

Fig. 5. Correlation of staining intensities of DNA lesions and asbestos contents. Asbestos contents and staining intensities of DNA lesions (8-nitroG and 8-oxodG) in lung tissues were evaluated and calculated as described in Materials and Methods. The correlation of asbestos contents and staining intensities of DNA lesions was analyzed by Pearson's correlation test. Black circle and gray triangle, subjects without $(\mathrm{n}=21)$ and with malignant mesothelioma $(n=15)$, respectively.

the amphibole content, although the correlation was not significant. When the fiber length was limited to $\geq 1 \mu \mathrm{m}$, better correlation with the staining intensity was observed (Table S2). The staining intensity of 8-nitroG was significantly correlated with the contents of total asbestos $(R=0.652, p=0.001)$ and amphiboles $(R=0.659, p=0.001)$. The staining intensity of APE1 was significantly correlated with the contents of total asbestos $(R=0.645, p=0.002)$ and amphiboles ( $R=0.619, p=0.003)$. The staining intensity of iNOS was significantly correlated with the contents of total fibers $(R=0.444, p=0.044)$ and amphiboles $(R=0.607$, $p=0.004)$. The staining intensities of these molecules were not significantly correlated with the contents of chrysotile and non-asbestos fibers. In mesothelioma patients, the staining intensities of these molecules 
Table 1. Multiple stepwise regression analysis of DNA lesions for subjects without mesothelioma $(n=21)$

(All fibers)

8-NitroG $\left(\mathrm{F}\right.$ value $=7.564$, adjusted $\left.\mathrm{R}^{2}=0.247, p=0.013\right)$

\begin{tabular}{|c|c|c|c|c|c|c|}
\hline & B & $95 \% \mathrm{CI}$ & SE & $\beta$ & $t$ & $p$ \\
\hline (Intercept) & -1.184 & $-2.953 \sim 0.585$ & 0.845 & & -1.401 & 0.177 \\
\hline Amphiboles & 0.382 & $0.091 \sim 0.673$ & 0.139 & 0.534 & 2.750 & $* 0.013$ \\
\hline \multicolumn{7}{|c|}{ 8-oxodG $\left(\mathrm{F}\right.$ value $=7.637$, adjusted $\left.\mathrm{R}^{2}=0.249, p=0.012\right)$} \\
\hline & B & $95 \% \mathrm{CI}$ & SE & $\beta$ & $t$ & $p$ \\
\hline (Intercept) & -0.992 & $-2.181 \sim 0.198$ & 0.568 & & -1.745 & 0.097 \\
\hline Age & 0.024 & $0.006 \sim 0.042$ & 0.009 & 0.535 & 2.764 & $* 0.012$ \\
\hline
\end{tabular}

(Fibers $\geq 1 \mu \mathrm{m})$

8-NitroG $\left(\mathrm{F}\right.$ value $=14.587$, adjusted $\left.\mathrm{R}^{2}=0.405, p=0.001\right)$

\begin{tabular}{|c|c|c|c|c|c|c|}
\hline & B & $95 \% \mathrm{CI}$ & SE & $\beta$ & $t$ & $p$ \\
\hline (Intercept) & -1.840 & $-3.474 \sim-0.205$ & 0.781 & & -2.356 & $* 0.029$ \\
\hline Amphiboles & 0.507 & $0.229 \sim 0.784$ & 0.133 & 0.659 & 3.819 & $* * 0.001$ \\
\hline \multicolumn{7}{|c|}{ 8-oxodG $\left(\mathrm{F}\right.$ value $=9.672$, adjusted $\left.\mathrm{R}^{2}=0.464, p=0.001\right)$} \\
\hline & B & $95 \% \mathrm{CI}$ & SE & $\beta$ & $t$ & $p$ \\
\hline (Intercept) & 1.376 & $-0.594 \sim 3.346$ & 0.938 & & 1.467 & 0.160 \\
\hline Age & 0.028 & $0.012 \sim 0.043$ & 0.007 & 0.632 & 3.788 & $* * 0.001$ \\
\hline Chrysotile & -0.475 & $-0.815 \sim-0.136$ & 0.162 & -0.491 & -2.939 & $* * 0.009$ \\
\hline
\end{tabular}

$\mathrm{B}$, unstandardized regression coefficient; $\mathrm{CI}$, confidence interval; $\mathrm{SE}$, standard error; $\beta$, standardized regression coefficient. ${ }^{*} p<0.05 ; * * p<0.01$.

were not significantly correlated with the contents of any types of fibers, except APE1 and non-asbestos fibers (Table S2).

Correlation of staining intensities with occupational asbestos exposure and age

We examined the correlation of staining intensities of DNA lesions and related molecules with total duration of occupational asbestos exposure and age. The staining intensity of 8-oxodG was significantly correlated with duration of occupational asbestos exposure in all cases $(R=0.487, p=0.003)$ and subjects without mesothelioma $(R=0.675, p=0.001)$ (Table S3). OGG1 expression was significantly correlated with duration of asbestos exposure in subjects without mesothelioma $(R=0.434, p=0.049)$. The staining intensities of the other molecules (8-nitroG, iNOS and APE1) had no significant correlation with duration of asbestos exposure (Table S3). The staining intensity of 8-oxodG was also significantly correlated with age in subjects without mesothelioma $(R=0.535, p=0.012)$ (Table S4). The staining intensity of OGG1 was significantly correlated with age in all cases $(R=0.463$, $p=0.004)$ and subjects without mesothelioma $(R=0.529$, $p=0.014$ ) (Table S4). The staining intensities of the other molecules were not significantly correlated with age.

\section{Multiple stepwise regression analysis}

We performed multiple stepwise regression analysis to determine which variables predict the extent of DNA damage. The analysis for all subjects revealed that none of the independent variables (contents of chrysotile, amphiboles and non-asbestos fibers, age and smoking habit) influenced the staining intensities of 8-nitroG and 8-oxodG. In subjects without mesothelioma, amphibole content was the only independent variable that influenced 8-nitroG formation $\left(R^{2}=0.247\right.$, $p=0.013$, Table 1). Age significantly influenced 8-oxodG formation $\left(R^{2}=0.249, p=0.012\right)$, but asbestos contents did not (Table 1). Regarding the fibers with a length of $\geq 1 \mu \mathrm{m}$, amphibole content was an independent variable for 8-nitroG formation $\left(R^{2}=0.405\right.$, $p=0.001$, Table 1). Age and chrysotile content positively $(\beta=0.632, p=0.001)$ and negatively $(\beta=-0.491$, $p=0.009$ ) influenced 8 -oxodG formation, respectively 
$\left(R^{2}=0.464, p=0.001\right.$, Table 1$)$, although it is unclear why chrysotile content was a negative determinant. In mesothelioma patients, no independent variable influenced the staining intensities of these molecules.

\section{Discussion}

In this study, we investigated the relationship between asbestos contents and the extent of DNA damage in human lung tissues. Immunohistochemical analysis revealed that 8-nitroG and 8-oxodG were formed in epithelial and inflammatory cells. Interestingly, in subjects without mesothelioma, the staining intensity of 8-nitroG was significantly correlated with the contents of total asbestos and amphiboles. This is the first study to clarify the association of 8-nitroG formation with asbestos contents in human lung tissues. When the fiber length was limited to $\geq 1 \mu \mathrm{m}$, there was a better correlation between 8 -nitroG formation and the contents of asbestos and amphiboles. This finding is in agreement with a number of studies demonstrating that length is a key factor in carcinogenicity and inflammogenicity of fibers ${ }^{17}$.

Carcinogenic fibers and particles induce an inflammatory response and the generation of ROS and RNS in respiratory systems ${ }^{11,18,19)}$. The roles of inflammatory responses and nitrative stress in asbestos-related diseases have been examined. iNOS expression and RNS generation have been observed in asbestostreated cultured human alveolar macrophages ${ }^{20)}$, lung epithelial cells ${ }^{21)}$ and mesothelial cells ${ }^{22)}$, and in the bronchiolar epithelium of asbestos-exposed animals ${ }^{23,24)}$. In clinical studies, the alveolar NO concentration was increased in asbestos-exposed subjects compared with control subjects ${ }^{25)}$. A recent study showed that the risk of asbestosis was associated with $i N O S$ gene polymorphism and increased with the number of CCTTT repeats, suggesting that iNOS activity is related to the pathogenesis of asbestos-related diseases ${ }^{26)}$. These findings suggest that nitrative stress plays an important role in the pathogenesis of asbestos-induced diseases and that nitratively modified biomolecules, including 8-nitroG, would be of clinical relevance in evaluation of asbestos exposure.

Amphiboles include iron-containing crocidolite and amosite, which are more potent carcinogens than chrysotile, which contains only a trace amount of iron as a contaminant ${ }^{27}$. Crocidolite has a much larger ability to cause DNA damage via iron-catalyzed ROS generation than chrysotile in a cell-free system ${ }^{27)}$. We demonstrated that crocidolite induced significantly stronger 8-nitroG formation than chrysotile in bronchial epithelial cells of mice ${ }^{11)}$. 8 -NitroG is formed by the interaction of guanine with $\mathrm{O}_{2}{ }^{--}$and $\mathrm{NO}^{7,8)}$. In addition, iNOS expression is mediated by the activation of the transcriptional factor $\mathrm{NF}-\kappa \mathrm{B}^{28)}$, which is activated by inflammatory cytokines and $\operatorname{ROS}^{29)}$. These findings suggest that ROS generation mediated by asbestos partially contributes to inflammatory reactions and the resulting 8-nitroG formation.

APE1 expression was significantly correlated with total asbestos and amphibole contents as well as 8-nitroG. APE1 was partially expressed in 8-nitroGpositive cells, and its staining intensity was significantly correlated with that of 8-nitroG. The glycosidic bond between 8-nitroG and deoxyribose is chemically unstable, and 8-nitroG can be spontaneously released to form an abasic site ${ }^{13)}$. These findings raise a possibility that 8-nitroG is partially removed from DNA and that the resulting abasic sites undergo APE1-mediated base excision repair. An unrepaired abasic site forms a pair with adenine during DNA synthesis, resulting in $\mathrm{G} \rightarrow \mathrm{T}$ transversion ${ }^{30)}$. $\mathrm{G} \rightarrow \mathrm{T}$ transversion may also occur via incorporation of adenine opposite 8-nitroG during DNA synthesis ${ }^{31,32)}$. This type of mutation was most frequently detected in the omentum of crocidolite-exposed rats ${ }^{33)}$. These observations support the formation of 8-nitroG in association with asbestos exposure.

In mesothelioma patients, the staining intensities of DNA lesions and repair enzymes were not correlated with asbestos contents in the lung. It is speculated that inflammatory reactions associated with mesothelioma development mediate additional oxidative/nitrative reactions to DNA lesions in lung tissues. Actually, various types of inflammatory cells infiltrate malignant mesothelioma tissues ${ }^{34)}$. Inflammatory molecules, including iNOS, cyclooxygenase- 2 and cytokines, are expressed in human mesothelioma cells ${ }^{35,36)}$. Under inflammatory conditions, various oxidizing agents, including $\mathrm{ONOO}^{-}$, are generated in biological systems to induce further oxidation of 8 -oxodG ${ }^{37,38)}$. 8-NitroG may undergo similar reactions during mesothelioma development. On the other hand, the asbestos contents in patients with mesothelioma were significantly higher than those in subjects without mesothelioma, and dose dependence of 8-nitroG formation may be lost at high asbestos contents. Therefore, under such conditions, the extent of DNA damage may not be correlated with asbestos contents.

It has been reported that the 8 -oxodG level increased in leukocytic DNA of asbestos-exposed workers with the grade of asbestosis ${ }^{39)}$. In this study, the staining intensity of 8-oxodG in lung tissues was not significantly correlated with asbestos contents but was correlated with duration of occupational asbestos exposure and age in subjects without mesothelioma. This finding is supported by an animal experiment showing that the 8-oxodG level in lung tissues of mice significantly increased with age ${ }^{40)}$. OGG1 expression was observed in 8-oxodG-positive cells and 
was significantly correlated with 8-oxodG formation and age. ROS are generated from multiple sources, including inflammatory cells, carcinogenic chemicals and the electron transport chain in mitochondria ${ }^{5)}$. Therefore, 8-oxodG formation may be affected by confounding factors other than asbestos. On the other hand, RNS are mainly generated under inflammatory conditions $^{5)}$. 8-NitroG staining was correlated with total asbestos and amphibole contents but not affected by confounding factors such as age and smoking habit.

The limitations of this study are as follows. 1) We mainly evaluated the staining intensity of DNA lesions in bronchial and alveolar epithelial cells. It was difficult to evaluate the extent of DNA damage in mesothelial cells of all specimens because some specimens did not contain enough of these cells for evaluation. 2) To analyze the relationship between DNA damage and asbestos contents, we included the cases of lung diseases, such as pneumonia and fibrosis, in subjects without mesothelioma. Therefore, we cannot exclude the possibility that histopathological changes in lung tissues, such as inflammatory reactions, influence the staining intensities of DNA lesions.

In conclusion, the findings of this study raise a possibility that 8-nitroG serves as a potential biomarker that can be used to evaluate asbestos exposure and carcinogenic risk. This study may provide insight into risk assessment of fibrous and particulate materials, including asbestos.

Acknowledgments: This work was supported by Grants-in-Aid for Scientific Research from the Ministry of Education, Culture, Sports, Science and Technology and the National Cancer Center Research and Development Fund in Japan.

\section{References}

1) IARC. Asbestos (chrysotile, amosite, crocidolite, tremolite, actinolite, and anthophyllite). In: IARC Monographs on the Evaluation of Carcinogenic Risks to Humans, vol. 100, A Review of Human Carcinogens Part C: Arsenic, Metals, Fibres, and Dusts. 2012. p. 219-309.

2) Robinson BW, Lake RA. Advances in malignant mesothelioma. N Engl J Med 2005; 353: 1591-603.

3) Coussens LM, Werb Z. Inflammation and cancer. Nature 2002; 420: 860-7.

4) Mossman BT, Lippmann M, Hesterberg TW, Kelsey KT, Barchowsky A, Bonner JC. Pulmonary endpoints (lung carcinomas and asbestosis) following inhalation exposure to asbestos. J Toxicol Environ Health B Crit Rev 2011; 14: 76-121.

5) Kawanishi S, Hiraku Y. Oxidative and nitrative DNA damage as biomarker for carcinogenesis with special reference to inflammation. Antioxid Redox
Signal 2006; 8: 1047-58.

6) Hussain SP, Hofseth LJ, Harris CC. Radical causes of cancer. Nat Rev Cancer 2003; 3: 276-85.

7) Kawanishi S, Hiraku Y, Pinlaor S, Ma N. Oxidative and nitrative DNA damage in animals and patients with inflammatory diseases in relation to inflammation-related carcinogenesis. Biol Chem 2006; 387: 365-72.

8) Yermilov V, Rubio J, Becchi M, Friesen MD, Pignatelli B, Ohshima H. Formation of 8-nitroguanine by the reaction of guanine with peroxynitrite in vitro. Carcinogenesis 1995; 16: 2045-50.

9) Hiraku Y, Tabata T, Ma N, Murata M, Ding X, Kawanishi S. Nitrative and oxidative DNA damage in cervical intraepithelial neoplasia associated with human papilloma virus infection. Cancer Sci 2007; 98: 964-72.

10) Ma N, Kawanishi M, Hiraku $Y$, et al. Reactive nitrogen species-dependent DNA damage in EBV-associated nasopharyngeal carcinoma: the relation to STAT3 activation and EGFR expression. Int J Cancer 2008; 122: 2517-25.

11) Hiraku Y, Kawanishi S, Ichinose T, Murata M. The role of iNOS-mediated DNA damage in infectionand asbestos-induced carcinogenesis. Ann NY Acad Sci 2010; 1203: 15-22.

12) Srinivasan A, Wang L, Cline CJ, et al. Identification and characterization of human apurinic/apyrimidinic endonuclease-1 inhibitors. Biochemistry 2012; 51: 6246-59.

13) Yermilov V, Rubio J, Ohshima H. Formation of 8-nitroguanine in DNA treated with peroxynitrite in vitro and its rapid removal from DNA by depurination. FEBS Lett 1995; 376: 207-10.

14) David SS, O'Shea VL, Kundu S. Base-excision repair of oxidative DNA damage. Nature 2007; 447: 941-50.

15) Sakai K, Hisanaga N, Huang J, et al. Asbestos and nonasbestos fiber content in lung tissue of Japanese patients with malignant mesothelioma. Cancer 1994; 73: $1825-35$.

16) Hiraku Y, Kawanishi S. Immunohistochemical analysis of 8-nitroguanine, a nitrative DNA lesion, in relation to inflammation-associated carcinogenesis. Methods Mol Biol 2009; 512: 3-13.

17) Donaldson K, Poland CA, Murphy FA, Macfarlane M, Chernova T, Schinwald A. Pulmonary toxicity of carbon nanotubes and asbestos-Similarities and differences. Adv Drug Deliv Rev 2013; 65: 2078-86.

18) Kawanishi $S$, Inoue $S$, Oikawa $S$, et al. Oxidative DNA damage in cultured cells and rat lungs by carcinogenic nickel compounds. Free Radic Biol Med 2001; 31: 108-16.

19) Mossman BT, Borm PJ, Castranova V, Costa DL, Donaldson K, Kleeberger SR. Mechanisms of action of inhaled fibers, particles and nanoparticles in lung and cardiovascular diseases. Part Fibre Toxicol 2007; 4: 4.

20) Quinlan TR, BeruBe KA, Hacker MP, et al. 
Mechanisms of asbestos-induced nitric oxide production by rat alveolar macrophages in inhalation and in vitro models. Free Radic Biol Med 1998; 24: 778-88.

21) Chao CC, Park SH, Aust AE. Participation of nitric oxide and iron in the oxidation of DNA in asbestostreated human lung epithelial cells. Arch Biochem Biophys 1996; 326: 152-7.

22) Riganti C, Orecchia S, Silvagno F, et al. Asbestos induces nitric oxide synthesis in mesothelioma cells via Rho signaling inhibition. Am J Respir Cell Mol Biol 2007; 36: 746-56.

23) Dorger M, Allmeling AM, Kiefmann R, Munzing $\mathrm{S}$, Messmer K, Krombach F. Early inflammatory response to asbestos exposure in rat and hamster lungs: role of inducible nitric oxide synthase. Toxicol Appl Pharmacol 2002; 181: 93-105.

24) Tanaka S, Choe N, Hemenway DR, Zhu S, Matalon $S$, Kagan E. Asbestos inhalation induces reactive nitrogen species and nitrotyrosine formation in the lungs and pleura of the rat. J Clin Invest 1998; 102: 445-54.

25) Lehtonen H, Oksa P, Lehtimaki L, et al. Increased alveolar nitric oxide concentration and high levels of leukotriene $\mathrm{B}_{4}$ and 8-isoprostane in exhaled breath condensate in patients with asbestosis. Thorax 2007; 62: 602-7.

26) Franko A, Dodic-Fikfak M, Arneric N, Dolzan V. Inducible nitric oxide synthase genetic polymorphism and risk of asbestosis. J Biomed Biotechnol 2011; 2011: 685870 .

27) Jiang L, Nagai $H$, Ohara $H$, et al. Characteristics and modifying factors of asbestos-induced oxidative DNA damage. Cancer Sci 2008; 99: 2142-51.

28) Karin M, Greten FR. NF- $\kappa$ B: linking inflammation and immunity to cancer development and progression. Nat Rev Immunol 2005; 5: 749-59.

29) Sethi G, Shanmugam MK, Ramachandran L, Kumar AP, Tergaonkar V. Multifaceted link between cancer and inflammation. Biosci Rep 2012; 32: 1-15.

30) Loeb LA, Preston BD. Mutagenesis by apurinic/ apyrimidinic sites. Annu Rev Genet 1986; 20: 201-30.

31) Suzuki N, Yasui M, Geacintov NE, Shafirovich V, Shibutani S. Miscoding events during DNA synthe- sis past the nitration-damaged base 8-nitroguanine. Biochemistry 2005; 44: 9238-45.

32) Bhamra I, Compagnone-Post P, O'Neil IA, Iwanejko LA, Bates AD, Cosstick R. Base-pairing preferences, physicochemical properties and mutational behaviour of the DNA lesion 8-nitroguanine. Nucleic Acids Res 2012; 40: 11126-38.

33) Unfried K, Schurkes C, Abel J. Distinct spectrum of mutations induced by crocidolite asbestos: clue for 8-hydroxydeoxyguanosine-dependent mutagenesis in vivo. Cancer Res 2002; 62: 99-104.

34) Suzuki K, Kadota K, Sima CS, et al. Chronic inflammation in tumor stroma is an independent predictor of prolonged survival in epithelioid malignant pleural mesothelioma patients. Cancer Immunol Immunother 2011; 60: 1721-8.

35) Marrogi A, Pass HI, Khan M, Metheny-Barlow LJ, Harris CC, Gerwin BI. Human mesothelioma samples overexpress both cyclooxygenase-2 (COX-2) and inducible nitric oxide synthase (NOS2): in vitro antiproliferative effects of a COX-2 inhibitor. Cancer Res 2000; 60: 3696-700.

36) Burt BM, Bader A, Winter D, Rodig SJ, Bueno R, Sugarbaker DJ. Expression of interleukin-4 receptor alpha in human pleural mesothelioma is associated with poor survival and promotion of tumor inflammation. Clin Cancer Res 2012; 18: 1568-77.

37) Niles JC, Wishnok JS, Tannenbaum SR. Peroxynitrite-induced oxidation and nitration products of guanine and 8-oxoguanine: structures and mechanisms of product formation. Nitric Oxide 2006; 14: 109-21.

38) Dizdaroglu M, Jaruga P. Mechanisms of free radical-induced damage to DNA. Free Radic Res 2012; 46: 382-419.

39) Takahashi K, Pan G, Kasai H, et al. Relationship between asbestos exposures and 8-hydroxydeoxyguanosine levels in leukocytic DNA of workers at a Chinese asbestos-material plant. Int J Occup Environ Health 1997; 3: 111-9.

40) Mikkelsen L, Bialkowski K, Risom L, Lohr M, Loft S, Moller P. Aging and defense against generation of 8-oxo-7,8-dihydro-2'-deoxyguanosine in DNA. Free Radic Biol Med 2009; 47: 608-15. 